\title{
HAUSDORFF-YOUNG INEQUALITIES FOR FUNCTIONS IN BERGMAN SPACES ON TUBE DOMAINS
}

\author{
by DAVID BEKOLLÉ and ALINE BONAMI
}

(Received 31st October 1996)

\begin{abstract}
We prove that the functions of the Bergman spaces $A^{p}$ on tube domains may be written as Laplace transforms of functions when $1 \leq p \leq 2$. We give in this context a generalization of the Hausdorff-Young inequality with the exact constant, and deduce from the case $p=2$ the expression of the Bergman kernel as a Laplace transform.
\end{abstract}

1991 Mathematics subject classification: 32A37.

\section{Introduction}

Let $\Gamma$ denote a non empty open connected proper subset of $\mathbb{R}^{n}$. Let $T_{\Gamma}$ be the tube over $\Gamma$,

$$
T_{\Gamma}=\left\{z=x+i y \in \mathbb{C}^{n} ; y \in \Gamma\right\}
$$

For every $p \in[1, \infty)$, the Bergman space $A^{p}\left(T_{\Gamma}\right)$ is defined as

$$
A^{p}\left(T_{\Gamma}\right)=L^{p}\left(T_{\Gamma}, d v\right) \cap \mathcal{H}\left(T_{\Gamma}\right)
$$

where $\mathcal{H}\left(T_{\Gamma}\right)$ is the space of holomorphic functions on $T_{\Gamma}$, and $d v$ is the Lebesgue measure on $\mathbb{C}^{n}$.

Our aim is to prove that all functions in $A^{p}\left(T_{\Gamma}\right)$, for $1 \leq p \leq 2$, are Laplace transforms of functions, and to give in this context a generalization of the usual Hausdorff-Young inequality.

Let us denote by $K=K_{\Gamma}$ the function on $\mathbb{R}^{n}$ which is defined by

$$
K(t)=\int_{\Gamma} e^{-2 \pi(t, u)} d u
$$

where $\langle t, u\rangle=\sum_{j=1}^{n} t_{j} u_{j}$ is the Euclidean scalar product. Then the main theorem is the following.

Theorem 1. Let $p$ belong to $[1,2]$, and let $p^{\prime}$ be the conjugate exponent of $p$. Then 
every $F \in A^{p}\left(T_{\Gamma}\right)$ is the Laplace transform of a unique function $f \in L^{p^{\prime}}\left(\mathbb{R}^{n}, K(p t)^{p^{\prime}-1} d t\right)$, namely

$$
F(z)=\int_{\mathbf{R}^{n}} f(t) e^{2 i \pi\{z, t\}} d t
$$

Moreover the map $F \rightarrow f$ is a bounded operator from $A^{p}\left(T_{\Gamma}\right)$ to $L^{p^{\prime}}\left(\mathbb{R}^{n}, K(p t)^{p^{\prime}-1} d t\right)$, with norm $\leq c_{p}$, that is, for $1<p \leq 2$

$$
\left[\int_{\mathbb{R}^{n}}|f(t)|^{p^{\prime}} K(p t)^{p^{\prime}-1} d t\right]^{1 / p^{\prime}} \leq c_{p}\left[\int_{T_{\Gamma}}|F(z)|^{p} d v(z)\right]^{1 / p}
$$

while, for $p=1$,

$$
\sup _{t \in \mathbf{R}^{n}}|f(t)| K(t) \leq \int_{T_{\Gamma}}|F(z)| d v(z)
$$

The constant $c_{p}$ may be taken equal to the best constant in the classical Hausdorff-Young inequality, that is

$$
c_{p}=\left[\frac{p^{1 / p}}{\left(p^{\prime}\right)^{1 / p^{\prime}}}\right]^{n / 2} .
$$

In the particular case when $p=2$, the operator $F \rightarrow f$ is unitary from $A^{2}\left(T_{\Gamma}\right)$ onto $L^{2}\left(\mathbb{R}^{n}, K(2 t) d t\right)$. So

$$
\int_{\mathbf{R}^{n}}|f(t)|^{2} K(2 t) d t=\int_{T_{\Gamma}}|F(z)|^{2} d v(z)
$$

The fact that the Laplace transform is well defined will be proved in the next section. We shall also prove that the constant of the classical Hausdorff-Young inequality is the best possible (see Theorem 5 below). So the norm of the operator $F \rightarrow f$ from $A^{p}\left(T_{\Gamma}\right)$ to $L^{p^{\prime}}\left(\mathbb{R}^{n}, K(p t)^{p^{\prime}-1} d t\right)$ is the same as the norm of the Fourier transform from $L^{p}\left(\mathbb{R}^{n}\right)$ to $L^{p^{\prime}}\left(\mathbb{R}^{n}\right)$. Remember that the best constant $c_{p}$ in the classical Hausdorff-Young inequality has been obtained by $\mathrm{W}$. Beckner [1].

Let us say briefly what was previously known in this area. In the case of the upper half-plane, the boundary value of a function $F \in A^{p}\left(T_{\Gamma}\right)$ belongs to the Besov space $\dot{B}_{p}^{-1 / p, p}(\mathbb{R})$ and the function $f$ is the Fourier transform of the boundary value of $F$. So (3) (with a bigger constant) follows from the characterization of the Besov spaces through Littlewood-Paley decomposition (see for instance [10]). For a general tube domain, the cases $p=1$ and $p=2$ have been obtained by T. Genchev [3]. Moreover T. Genchev has given some partial results for $1<p<2$ for starlike cones [4]. The problem of obtaining a generalization of the classical Hausdorff-Young inequality has 
also been considered in [5]. But none of these two papers contains the boundedness of the operator $F \rightarrow f$ from $A^{p}\left(T_{\Gamma}\right)$ to $L^{p^{\prime}}\left(\mathbb{R}^{n}, K(p t)^{p^{\prime}-1} d t\right)$ when $1<p<2$.

Let us remark that it follows from Theorem 1 that $A^{P}\left(T_{\Gamma}\right)=\{0\}$ whenever $K(t)=+\infty$ a.e. As we shall see, the reverse assertion is also valid. So, as a corollary, we get:

Theorem 2. Let $p$ belong to $[1, \infty)$. Then $A^{p}\left(T_{\Gamma}\right)=\{0\}$ if and only if $K(t)=+\infty$ a.e.

When $\Gamma$ is convex, this condition is equivalent to the fact that $\Gamma$ does not contain any straight line. When $\Gamma$ is a cone, it is equivalent to the fact that $\Gamma^{*}$ is not void, where $\Gamma^{*}$ denotes the open dual cone of $\Gamma$, given by

$$
\Gamma^{*}=\left\{t \in \mathbb{R}^{n} ;\langle t, y\rangle>0, y \in \bar{\Gamma} \backslash\{0\}\right\}
$$

Moreover, in this case the function $f$ given in Theorem 1 is 0 a.e. outside $\Gamma^{*}$.

Bochner's theorem asserts that the tube domains $T_{\Gamma}$ are domains of holomorphy if and only if $\Gamma$ is convex (see [6]). Moreover, the envelope of holomorphy of $T_{\Gamma}$ is $T_{\Gamma}$, where $\Gamma^{c}$ is the convex hull of $\Gamma$. So $\mathcal{H}\left(T_{\Gamma}\right) \equiv \mathcal{H}\left(T_{\Gamma^{c}}\right)$. The same equivalence holds for Hardy spaces as it is shown in [9]. It is no more the case for Bergman spaces. We give counter-examples, which are based on the following corollary of Theorem 1.

Theorem 3. The restriction operator from $A^{2}\left(T_{\Gamma c}\right)$ to $A^{2}\left(T_{\Gamma}\right)$ is onto if and only if there exists a constant $C$ such that, for almost every $t \in \mathbb{R}^{n}$,

$$
\int_{\Gamma^{e}} e^{-2 \pi(t, u)} d u \leq C \int_{\Gamma} e^{-2 \pi(t, u)} d u
$$

When $\Gamma^{c}$ is a homogeneous cone, D. Luecking (see [8]) has given a necessary and sufficient condition of a different nature, which implies that all the Bergman spaces on $T_{\Gamma}$ and $T_{\Gamma}$ respectively are the same for every value of $p$.

We shall proceed as follows. We prove Theorem 1 in Section 2. In Section 3, we give Laplace transforms formulae for the Bergman kernel of $T_{\Gamma}$. Such formulae are known in the case of convex cones (see [7]). We also give formulae for the holomorphic continuation $\tilde{F}$ of a function $F \in A^{p}\left(T_{\Gamma}\right), 1 \leq p \leq 2$. We prove Theorem 3 at the end of this section, as well as the counter-examples.

Finally, let us remark that the interpolation space between $A^{1}$ and $A^{2}$ is $A^{p}$ when $\Gamma$ is a homogeneous cone. It is a consequence of the fact that there exists a bounded projection $\Pi$ from $L^{\prime}\left(T_{\Gamma}\right)$ to $A^{\prime}\left(T_{\Gamma}\right)$ which is also bounded on $L^{2}\left(T_{\Gamma}\right)$. This last fact is an easy corollary of the results of [2]. We write it here for completeness.

Theorem 4. When $\Gamma$ is a homogeneous cone, then the interpolation space

$$
\left(A^{1}\left(T_{\Gamma}\right), A^{2}\left(T_{\Gamma}\right)\right)_{0}
$$

is isomorphic to $A^{p}\left(T_{\Gamma}\right)$ for $p=2 /(\theta+1)$. 
Proof. If $B$ denotes the Bergman kernel, the operator $\Pi$ of kernel $[B(z, \zeta)]^{2} B(\zeta, \zeta)^{-1}$ has the required properties (see [2]).

In this case, (3) in Theorem 1 (with a bigger constant) follows by interpolation between the cases $p=1$ and $p=2$, which is the usual proof of the classical HausdorffYoung inequalities.

\section{Laplace transforms and Hausdorff-Young inequalities}

Our first proposition deals with Laplace transforms. We write $F=\mathcal{L}(f)$ when $F$ is the Laplace transform of $f$, that is:

$$
F(z)=\int_{\mathbf{R}^{\mathbf{n}}} f(t) e^{2 i \pi(z, t)} d t
$$

Of course, $F$ is well defined only when $f$ is sufficiently decreasing at $\infty$. The function $K=K_{\mathrm{r}}$ has been defined in (1).

Proposition 2.1. Assume that $p \in(1, \infty]$. Let $g$ be a measurable function such that:

$$
\begin{array}{cc}
\int|g(t)|^{p} K\left(p^{\prime} t\right)^{p-1} d t<\infty & \text { if } p<\infty \\
\sup \{|g(t)| K(t)\}<\infty & \text { if } p=\infty
\end{array}
$$

Then, for every $y \in \Gamma$, the function $t \mapsto g(t) e^{-2 \pi(y, t)}$ is integrable, and so the Laplace transform $\mathcal{L}(g)$ is well defined. Moreover it is a holomorphic function in $T_{\Gamma}$, and the following estimates are valid:

$$
\int_{T_{\Gamma}}|\mathcal{L}(g)(z)|^{2} d v(z)=\int|g(t)|^{2} K(2 t) d t
$$

For $1<p<2$,

$$
\left[\int_{T_{\Gamma}}|\mathcal{L}(g)(z)|^{p^{\prime}} d v(z)\right]^{1 / p^{\prime}} \leq c_{p}\left[\int_{\mathbf{R}^{n}}|g(t)|^{p} K\left(p^{\prime} t\right)^{p-1} d t\right]^{1 / p} .
$$

Proof. Let us first consider a particular case. Let $P$ be the product of intervals $P=(-1,+1)^{n}$. Then

$$
K_{P}(t)=\prod_{j=1}^{n} \frac{\sinh \left(2 \pi t_{j}\right)}{\pi t_{j}}
$$


Let us show in this case that $\mathcal{L}(g)$ is well defined and holomorphic on $T_{P}$. For $a \in(0,1)$, let us write $P_{a}=(-a,+a)^{n}$. To prove that the function is holomorphic in the interior of $T_{P_{a}}$ it is sufficient to show that $g(t) \prod_{j=1}^{n} \cosh \left(2 \pi a t_{j}\right)$ is integrable in $\mathbb{R}^{n}$. Using the Hölder inequality and the hypothesis on $g$, it follows from the inequality

$$
\int_{\mathbf{R}^{n}}\left[\prod_{j=1}^{n} \cosh \left(2 \pi a t_{j}\right)\right]^{p^{\prime}} K_{P}\left(p^{\prime} t\right)^{-1} d t<\infty
$$

which is an easy consequence of the expression of $K_{P}$. When $Q$ is any product of intervals, the same result is obtained from the result for $P$ using translation and dilation. Let us now consider the general case. Let $Q$ denote a product of intervals which is contained in $\Gamma$. Then $K_{Q} \leq K_{\Gamma}$, and $g$ satisfies also the hypothesis with $Q$ instead of $\Gamma$. So $\mathcal{L}(g)$ is well defined and holomorphic on $T_{Q}$. As this is valid for each $Q, \mathcal{L}(g)$ is well defined and holomorphic on $T_{\Gamma}$.

To prove (7), we use the Plancherel identity. To prove (8), we use the classical Hausdorff-Young inequality to get

$$
\int_{T_{\Gamma}}|\mathcal{L}(g)(z)|^{p^{\prime}} d v(z) \leq\left(c_{p}\right)^{p^{\prime}} \int_{\Gamma}\left[\int_{\mathbf{R}^{n}}|g(t)|^{p} e^{-2 p \pi(y, t)} d t\right]^{p^{\prime} / p} d y .
$$

We then use Minkowski inequality for the $L^{p^{\prime} / p}$ norm, and the definition of $K$ to conclude.

Let us remark that inequality (8) may be seen as the dual version of the Hausdorff-Young inequality (3).

As a consequence of Proposition 2.1, we see that all Bergman spaces contain non zero functions when $K(t)<\infty$ on a set of positive measure: it follows directly from (8) for $A^{p}\left(T_{\Gamma}\right)$ when $p \geq 2$. For other values of $p$, just remark that if $A^{2 p}\left(T_{\Gamma}\right) \neq\{0\}$ then the same is true for $A^{p}\left(T_{\Gamma}\right)$ : if $F$ belongs to the first one of these spaces, then $F^{2}$ belongs to the second one. So we have proved one implication in Theorem 2. The other one is a direct consequence of Theorem 1 for $p \leq 2$ : if $K(t)=\infty$ a.e., then $f \equiv 0$ for each $F \in A^{p}\left(T_{\Gamma}\right)$, and so $A^{p}\left(T_{\Gamma}\right)=\{0\}$. For other values of $p$, let us use again that if $A^{2 p}\left(T_{\Gamma}\right) \neq\{0\}$ then the same is true for $A^{p}\left(T_{\Gamma}\right)$. An easy induction gives all values of $p$ from the values in $[1,2]$.

To write every $F \in A^{p}\left(T_{\Gamma}\right)$ as a Laplace transform, we first prove the following lemma using a classical method of Hardy. It is also given in [4] in this context.

Lemma 2.1. Let $p \in[1, \infty)$. For every $F \in A^{p}\left(T_{\Gamma}\right)$ and every $y \in \Gamma$, the function $x \mapsto F(x+i y)$ belongs to the space $L^{p}\left(\mathbb{R}^{n}\right)$.

Proof. Let us denote by $d(y)$ the Euclidean distance from $y$ to $\partial \Gamma$, and by $B(x+i y)$ the Euclidean open ball with centre $x+i y$ and radius $d(y) / 2$. Then $B(x+i y) \subset T_{\Gamma}$; moreover, by the mean value formula and by the Hölder inequality, 


$$
|F(x+i y)|^{p} \leq \frac{C_{p}}{[d(y)]^{2 n}} \int_{B(x+i y)}|F(\omega)|^{p} d v(\omega) \leq \frac{C_{p}}{[d(y)]^{2 n}} \int_{\mid x-\text { Rew|<d(y) }}|F(\omega)|^{p} d v(\omega) .
$$

Then the use of the Fubini theorem yields that

$$
\int_{\mathbf{R}^{n}}|F(x+i y)|^{p} d x \leq \frac{C_{p}}{(d(y))^{n}} \int_{T_{\mathbf{r}}}|F(\omega)|^{p} d v(\omega)
$$

We also have, for $q>p$,

$$
\int_{\mathbf{R}^{n}}|F(x+i y)|^{q} d x \leq \frac{C_{p q}}{(d(y))^{n\left(\frac{2 q}{p}-1\right)}}\left(\int_{T_{\Gamma}}|F(\omega)|^{p} d v(\omega)\right)^{q / p} .
$$

Let us now prove Theorem 1. By Lemma 2.1 and by the usual Hausdorff-Young inequality we know that, for every $F \in A^{p}\left(T_{\Gamma}\right)$ and for every $y \in \Gamma$, the function $x \mapsto F(x+i y)$ has a Fourier transform which is in $L^{p^{\prime}}\left(\mathbb{R}^{n}\right)$. We write it as

$$
f_{y}(t) e^{-2 \pi(y, t)}=\int_{\mathbf{R}^{n}} F(x+i y) e^{-2 i \pi(x, t)} d x
$$

We shall prove that the function $f_{y}$ is independent of $y$. To do this, we use the fact that such a property is valid when $F$ belongs to a Hardy space (see [9, p. 100). More precisely, since $\Gamma$ is connected, it is sufficient to prove that, for every fixed $y \in \Gamma, f_{y}(t)=f_{y^{\prime}}(t)$ almost everywhere when $y^{\prime}$ belongs to some neighbourhood of $y$. Let us choose $B=B(y, d(y) / 2)$ as such a neighbourhood. Then $F$ belongs to the Hardy space $H^{2}\left(T_{B}\right)$ by (10). We conclude using the result for $H^{2}$ spaces.

The function $f=f_{y}$ for some (all) $y \in \Gamma$ is the function that we were looking for. It satisfies

$$
f(t) e^{-2 \pi(y, t)}=\int_{\mathbf{R}^{n}} F(x+i y) e^{-2 i \pi(x, t)} d x
$$

Here the right hand side stands for the Fourier transform of a function in $L^{p}\left(\mathbb{R}^{n}\right)$. In particular for $1<p \leq 2$, by the Hausdorff-Young inequality,

$$
\left[\int|f(t)|^{p^{\prime}} e^{-2 p^{\prime} \pi(y, t)} d t\right]^{1 / p^{\prime}} \leq c_{p}\left[\int_{\mathbf{R}^{n}}|F(x+i y)|^{p} d x\right]^{1 / p} .
$$

Let us prove that $f$ satisfies the inequality (3). Let

$$
I=\left[\int_{\mathbf{R}^{n}}|f(t)|^{p^{\prime}} K(p t)^{p^{\prime}-1} d t\right]^{1 /\left(p^{\prime}-1\right)}=\left[\int_{\mathbf{R}^{n}}\left(\int_{\Gamma}|f(t)|^{p} e^{-2 \pi p(t, y)} d y\right)^{p^{\prime}-1} d t\right]^{1 /\left(p^{\prime}-1\right)} .
$$


By the Minkowski inequality used for the $L^{p^{\prime}-1}$ norm, we have

$$
I \leq \int_{\Gamma}\left(\int_{\mathbf{R}^{n}}|f(t)|^{p^{\prime}} e^{-2 \pi p^{\prime}(t, y)} d t\right)^{1 /\left(p^{\prime}-1\right)} d y .
$$

Using (12), we find the following inequality:

$$
I \leq\left(c_{p}\right)^{p} \int_{\Gamma}\left(\int_{\mathbf{R}^{n}}|F(x+i y)|^{p} d x\right) d y .
$$

The right hand side is exactly the integral in the tube domain $T_{\Gamma}$. This proves (3). We know from Proposition 2.1 that the Laplace transform of $f$ is well defined. Moreover, by the inverse Fourier transform formula, it is equal to $F$. We have finished the proof of Theorem 1 for $p \in(1,2)$. For $p=1$ the proof follows the same lines, and the inequality is obtained directly. For $p=2$ the equality in (5) follows from Plancherel identity, and the fact the map is onto is a consequence of Proposition 2.1.

Let us restrict to the case of convex domains in the following proposition.

Proposition 2.2. When $\Gamma$ is convex, then $A^{p}\left(T_{\Gamma}\right)=\{0\}$ for some $p>1$ if and only if $\Gamma$ contains a straight line.

Proof. Let $\Gamma$ contain a straight line. Using a rotation and translation if necessary, we may assume that it is the $y_{1}$ axis. As $\Gamma$ is open and convex, it contains also a cylinder $C_{\varepsilon}$, with

$$
C_{\varepsilon}=\left\{y \in \mathbb{R}^{n} ;\left|y_{2}\right|^{2}+\ldots+\left|y_{n}\right|^{2}<\varepsilon^{2}\right\}
$$

It follows that $K(t)=+\infty$ for every $t$. By Theorem $2, A^{p}\left(T_{\Gamma}\right)=\{0\}$. Assume now that $\Gamma$ contains no straight line. Then $\Gamma$ is contained in an open convex cone which contains no straight line (see [9]). For such a cone $\Gamma_{0}$, which we may assume issued from 0 , there exists $t_{0} \in \mathbb{R}^{n}$ and $\varepsilon_{0}>0$ such that $\left\langle t_{0}, y\right\rangle>\varepsilon_{0}|y|$ for every $y \in \Gamma_{0}$. It follows that $K(t)<\infty$ in a neighbourhood of $t_{0}$, and so $A^{p}\left(T_{\Gamma}\right) \neq\{0\}$.

Let us now restrict to cones.

Proposition 2.3. When $\Gamma$ is a cone, then $A^{p}\left(T_{\Gamma}\right)=\{0\}$ for some $p>1$ if and only if $\Gamma^{*}$ is void. Assume that $\Gamma^{*}$ is non void. Then every $F \in A^{p}\left(T_{\Gamma}\right)$ is the Laplace transform of a function which is zero a.e. outside $\Gamma^{*}$.

Proof. Let us denote by $\Gamma^{\#}$ the closed dual cone of $\Gamma$

$$
\Gamma^{\#}=\left\{t \in \mathbb{R}^{n} ;(t, y\rangle \geq 0, y \in \bar{\Gamma}\right\} .
$$


We shall use the following lemma.

Lemma 2.2. $\Gamma^{*}$ is the interior of $\Gamma^{\#}$. Moreover, when $\Gamma^{*}=\emptyset$, then $\Gamma^{\#}$ is contained in a hyperplane; when $\Gamma^{*} \neq \emptyset$. then $\Gamma^{\#}$ is the closure of $\Gamma^{*}$, and $\Gamma^{\#} \backslash \Gamma^{*}$ has Lebesgue measure 0.

Proof. The first assertion is a direct consequence of the definitions. To prove the second one, just remark that $\Gamma^{\#}$ is a convex cone. If it is not contained in a hyperplane, then it contains the convex hull of a union of $n$ half-lines which are generated by $n$ independent vectors; hence its interior is non void. Next, assume that $\Gamma^{*} \neq \emptyset$. Clearly $\Gamma^{\#}$ contains the closure of $\Gamma^{*}$. Conversely, by convexity, if $t$ belongs to $\Gamma^{\#}$ then the set

$$
\left\{t+\lambda B\left(t_{0}, \varepsilon\right), \lambda \in[0,1]\right\}
$$

is contained in $\Gamma^{\#}$. Here $B\left(t_{0}, \varepsilon\right)$ is any ball in $\Gamma^{*}$. So $t$ belongs to the closure of $\Gamma^{*}$. Now it is well known that, as $\Gamma^{*}$ is a convex open cone, $\Gamma^{*} \backslash \Gamma^{*}$ has Lebesgue measure 0 .

To prove Proposition 2.3, it is sufficient to prove that $K(t)<+\infty$ for $t \in \Gamma^{*}$, while $K(t)=+\infty$ for $t \notin \Gamma^{\#}$. This is a consequence of the following lemma:

Lemma 2.3. For $t \in \Gamma^{*}$, there exists $\varepsilon>0$ such that, for $y \in \Gamma\langle t, y\rangle \geq \varepsilon|y|$. For $t \notin \Gamma^{\#}$, there exists $\varepsilon>0$ and an open non void cone $\Gamma_{0}$ contained in $\Gamma$ such that, for $y \in \Gamma_{0},\langle t, y\rangle \leq-\varepsilon|y|$.

Remark 2.1. On $\Gamma^{\sharp} \backslash \Gamma^{*}, K$ may be finite or not. In [7], it is proved that $K=+\infty$ when $\Gamma$ is a convex cone. Our example in Section 3 is based on an example of a cone $\Gamma$ for which $K$ is not identically $+\infty$ on $\partial \Gamma^{*}$.

Finally, let us prove that the constant $c_{p}$ is the best possible. More precisely, let us prove the following theorem.

Theorem 5. Let $\Gamma$ be an open connected set in $\mathbb{R}^{n}$ such that $A^{p}\left(T_{\Gamma}\right) \neq\{0\}$. Then the smallest constant $c_{p}$ such that every $F \in A^{p}\left(T_{\Gamma}\right)$ may be written as the Laplace transform of $f$, with

$$
\left[\int_{\mathbf{R}^{n}}|f(t)|^{p^{\prime}} K(p t)^{p^{\prime}-1} d t\right]^{1 / p^{\prime}} \leq c_{p}\left[\int_{T_{\Gamma}}|F(z)|^{p} d v(z)\right]^{1 / p}
$$

is equal to $\left[\frac{p^{1 / p}}{\left(p^{\prime}\right)^{1 / p^{\prime}}}\right]^{n / 2}$.

Proof. The set $E=\left\{t \in \mathbb{R}^{n} ; K(t)<\infty\right\}$ is convex and has positive measure. Hence 
it contains a ball. We shall call $p t^{0}$ its centre. From the inequality

$$
e^{2 \pi|| u \mid} \leq \prod_{j=1}^{n}\left(e^{2 \pi \varepsilon u_{j}}+e^{-2 \pi \varepsilon u_{j}}\right)
$$

it follows that

$$
\int_{\Gamma} e^{-2 \pi\left(p 0^{0}, u\right)} e^{2 \pi \varepsilon|u|} d u \leq \sum K\left(p t^{0}+\varepsilon\left(\eta_{1}, \eta_{2} \cdots \eta_{n}\right)\right)
$$

where the sum is taken on all $\eta_{j} \in\{-1,+1\}$. So, for $\varepsilon$ small enough,

$$
\int_{\Gamma} e^{-2 \pi\left(p r^{0}, u\right)} e^{2 \pi \varepsilon|u|} d u \leq<\infty .
$$

Assume that (13) is valid with the constant $c_{p}$. We shall prove that the usual Hausdorff-Young inequality is also valid with the same constant $c_{p}$, that is

$$
\left[\int_{\mathrm{R}^{n}}|\hat{g}(t)|^{p^{\prime}} d t\right]^{1 / p^{\prime}} \leq c_{p}\left[\int_{\mathrm{R}^{n}}|g(x)|^{p} d x\right]^{1 / p}
$$

for every function $g \in L^{p}\left(\mathbb{R}^{n}\right)$. Here $\hat{g}$ is the Fourier transform of $g$, given by

$$
\hat{g}(t)=\int_{\mathbf{R}^{n}} g(x) e^{-2 i \pi(x, t)} d x .
$$

From density arguments and dilation, it is sufficient to prove (15) when $\hat{g}$ is of class $\mathcal{C}^{\infty}$ and supported in the unit ball. Let us consider

$$
F_{N}(z)=N^{n / p^{\prime}} \int_{\mathbf{R}^{n}} \hat{g}\left(N\left(t-t^{0}\right)\right) e^{2 i \pi(z, t)} d t .
$$

The left hand side of (13), written for $F_{N}$, gives

$$
\left[\int_{\mathbf{R}^{n}}|\hat{g}(t)|^{p^{\prime}} K\left(p t^{0}+p N^{-1} t\right)^{p^{\prime}-1} d t\right]^{1 / p^{\prime}},
$$

which tends to

$$
K\left(p t^{0}\right)^{1 / p}\left[\int_{\mathbb{R}^{\prime \prime}}|\hat{g}(t)|^{p^{\prime}} d t\right]^{1 / p^{\prime}}
$$

The right hand side of (13), written again for $F_{N}$, is equal to 


$$
c_{p} N^{n / p}\left[\int_{\Gamma} \int_{\mathbf{R}^{n}}\left|F_{N}(N x+i y)\right|^{p} d x d y\right]^{1 / p} .
$$

But

$$
N^{n / p} F_{N}(N x+i y)=e^{2 i \pi\left(N x+i y, l^{0}\right)} \int_{\mathbf{R}^{n}} \hat{g}(t) e^{2 i \pi\left(x+i \frac{y}{N}, t\right\rangle} d t
$$

It follows from a theorem of Paley-Wiener (see [11]) that there exists some constant $C$ such that

$$
\left|N^{n / p} F_{N}(N x+i y)\right| \leq C(1+|x|)^{-2 n} e^{-2 \pi\left(y, t^{0}\right)} e^{\left|\frac{y}{N}\right|}
$$

while $N^{n / p}\left|F_{N}(N x+i y)\right|$ tends to $e^{-2 \pi\left(y, t^{0}\right)}|g(x)|$. Using (14), we can apply the theorem of dominated convergence. The right hand side of (13) tends to

$$
c_{p} K\left(p t^{0}\right)^{1 / p}\left[\int_{\mathrm{R}^{n}}|g(x)|^{p} d x\right]^{1 / p},
$$

which gives (15) and finishes the proof.

Remark 2.2. The same kind of proof allows to show that

$$
\left[\frac{p^{1 / p}}{\left(p^{\prime}\right)^{1 / p^{\prime}}}\right]^{n / 2}
$$

is also the best constant in (8).

3. Bergman kernel and holomorphic continuation to the convex hull

In this section, we give some applications of Theorem 1.

\subsection{Bergman kernel of a tube domain}

We prove that the Bergman kernel may be written as a Laplace transform.

Corollary 3.1. Let $\Gamma$ be a non empty open connected subset of $\mathbb{R}^{n}$ such that $K(t)<\infty$ on a set of positive measure. Then the Bergman kernel $B(z, \zeta)$ of $T_{\Gamma}$ is given by

$$
B(z, \zeta)=\int_{\mathbf{R}^{n}}(K(2 t))^{-1} e^{2 \pi i(z-\bar{\zeta}, t)} d t \quad z, \zeta \in T_{\Gamma}
$$


Here, by definition, $(K(t))^{-1}$ is taken equal to 0 when $K(t)=+\infty$.

Proof. The Bergman kernel $B(z, \zeta)$ is characterized by the following three properties:

(i) for every $z, \zeta \in T_{\mathrm{r}}$,

$$
B(\zeta, z)=\overline{B(z, \zeta)}
$$

(ii) for every $\zeta \in T_{\mathrm{r}}$, the function $B(., \zeta)$ belongs to $A^{2}\left(T_{\Gamma}\right)$;

(iii) for every $F \in A^{2}\left(T_{\Gamma}\right)$ and for every $z \in T_{\Gamma}$

$$
F(z)=\int_{T_{\Gamma}} B(z, \zeta) F(z) d v(z)
$$

Let us prove (ii). By Theorem 1, it is sufficient to show that

$$
\int_{\mathbf{R}^{n}}(K(2 t))^{-1} e^{-4 \pi\{\eta, t)} d t<\infty
$$

for all $\eta \in \Gamma$. Let us first remark that the set $E$ of $\eta \in \mathbb{R}^{n}$ such that (17) holds is a convex set. So if it contains almost every point of a product of intervals, called again $Q$, then it contains $Q$ itself. We shall use this property and prove that $E$ contains every product of intervals which is contained in $\Gamma$. More precisely, using dilation and translation if necessary, we assume that $P$ is contained in $\Gamma$ and prove that $P_{a}$ is contained in $E$ for $a \in(0,1)$. To do this, it is sufficient to show that

$$
\int_{P_{a}} \int_{\mathbf{R}^{n}}(K(2 t))^{-1} e^{-4 \pi(\eta, t)} d t d \eta<\infty
$$

But this integral is equal to

$$
\int_{\mathbf{R}^{n}}(K(2 t))^{-1} K_{P_{a}}(2 t) d t
$$

Let us use the fact that $K_{P} \leq K_{\Gamma}=K$, and the expression of $K_{p}$. We find that

$$
\int_{\mathbf{R}^{n}}(K(2 t))^{-1} K_{P_{a}}(2 t) d t \leq\left(\int_{-\infty}^{+\infty} \frac{\sinh (4 \pi a t)}{\sinh (4 \pi t)} d t\right)^{n}<\infty
$$

for $a<1$, which allows to conclude. Let us prove (iii). Let $F \in A^{2}\left(T_{\Gamma}\right)$. Then, by Theorem $1, F=\mathcal{L} f$ with $f \in L^{2}\left(\mathbb{R}^{n}, K(2 t) d t\right)$. In view of properties (i) and (ii), for $z \in T_{\Gamma}$,

$$
\int_{T_{\Gamma}} B(z, \zeta) F(\zeta) d v(\zeta)=\langle F, B(., z)\rangle_{A^{2}\left(T_{\Gamma}\right)}=\int_{\mathbf{R}^{n}} f(t) e^{2 \pi i(z, t)} d t=F(z)
$$

We used the polarization of (5). This proves the Corollary 3.1 . 
Let us give some of the consequences of this explicit formula. Let us first remark that

$$
|B(z, \zeta)| \leq(B(z, z))^{1 / 2}(B(\zeta, \zeta))^{1 / 2} .
$$

One has also the following inequality for cones, namely

$$
|B(z, \zeta)| \leq 2^{2 n} B(z, z)
$$

To prove (18), we write

$$
B(z, \zeta)=\int_{\Gamma^{*}}(K(2 t))^{-1} e^{2 \pi i(z-\bar{\zeta}(t)} d t
$$

It follows that

$$
|B(z, \zeta)| \leq \int_{\Gamma^{*}}(K(2 t))^{-1} e^{-2 \pi(y, t)} d t=2^{2 n} \int_{\Gamma^{*}}(K(2 t))^{-1} e^{-4 \pi(y, t)} d t,
$$

the last equality coming from homogeneity.

\subsection{Analytic continuation to the convex hull}

Let $\Gamma^{c}$ be the convex hull of the open subset $\Gamma$. From Bochner's Theorem we know that each holomorphic function on $T_{\Gamma}$ extends holomorphically to $T_{\Gamma}$. We prove now that for Bergman spaces the extension is given as a Laplace transform. The next proposition is a refinement of Proposition 2.1.

Proposition 3.1. Assume that $p \in[1, \infty]$. Let $g$ be a measurable function such that:

$$
\begin{array}{cc}
\int|g(t)|^{p} K\left(p^{\prime} t\right)^{p-1} d t<\infty & \text { if } p<\infty \\
\sup \{|g(t)| K(t)\}<\infty & \text { if } p=\infty .
\end{array}
$$

Then, for every $y \in \Gamma^{c}$, the function $t \mapsto g(t) e^{-2 \pi(y, t)}$ is integrable, and so the Laplace transform $\mathcal{L}(g)$ is well defined. Moreover it is a holomorphic function in $T_{\Gamma}$

Proof. From the proof of Proposition 2.1 it follows that, when $y^{0}$ belongs to $\Gamma$, then $g(t) e^{-2 \pi(y, n)}$ is bounded by an integrable function of $t$ for $y$ in a neighbourhood of $y^{0}$. Let now $y^{0}$ belong to $\Gamma^{c}$. We can write $y^{0}=\sum_{j=1}^{N} \lambda_{j} y^{j}$, with $y^{1}, \ldots, y^{N}$ in $\Gamma$ and $\sum_{j=1}^{N} \lambda_{j}=1$. By convexity of the exponential function,

$$
|g(t)| e^{-2 \pi\left(y^{0}, n\right)} \leq \sum_{j=1}^{N} \lambda_{j}|g(t)| e^{-2 \pi\left(y^{\prime}, t\right)}
$$


which gives the integrability of $|g(t)| e^{-2 \pi\left(y^{0} . t\right)}$. The same proof gives the boundedness by an integrable function in a neighbourhood of $y^{0}$.

Let us remark that in particular the extension of the Bergman kernel $T_{\Gamma}$ to $T_{\Gamma}$ is also given by Formula (16). We still use the notation $B(z, \zeta)$ for the extension. Moreover, with the same modification of the proof of Corollary 3.1 as in the proof of the last proposition, one can show that, for every $\zeta \in T_{\Gamma^{c}}$, the function $B(., \zeta)$ is in the space $A^{2}\left(T_{\Gamma}\right)$. As a consequence, we get the following.

Proposition 3.2. Let $F$ be a function in $A^{2}\left(T_{\Gamma}\right)$, and let $\tilde{F}$ be its extension to $A^{2}\left(T_{\Gamma^{c}}\right)$. Then, for $z \in T_{\Gamma^{c}}$,

$$
\tilde{F}(z)=\int_{T_{\Gamma}} B(z, \zeta) F(\zeta) d v(\zeta)
$$

Let us now give the proof of Theorem 3. Let us write $K_{c}(t)=\int_{\Gamma^{c}} e^{-2 \pi(t, u)} d u$. Then, using Theorem 1, it is clear that the inequality $K_{c} \leq C K$ and the Proposition 3.1 have as a consequence that the extension of each function in $A^{2}\left(T_{\Gamma}\right)$ belongs to $A^{2}\left(T_{\Gamma}\right)$. Conversely, assume that the restriction is onto. Then, using the closed graph theorem and Theorem 1, we know that there exists a constant $C$ such that for each positive function $f$

$$
\int_{\Gamma^{c}}|f(t)|^{2} K_{c}(t) d t \leq C \int_{\Gamma}|f(t)|^{2} K(t) d t
$$

The inequality $K_{c} \leq C K$ follows at once.

Let us give counter-examples for which the Bergman spaces of $T_{\Gamma}$ and $T_{\Gamma}$ do not coincide. Let us first give an example in two dimensions for which the first ones contain non zero functions while the second ones do not. Take

$$
\Gamma=\left\{y=\left(y_{1}, y_{2}\right) ; 0<y_{1}<\exp \left(-y_{2}^{2}\right)\right\}
$$

Then $\Gamma^{c}=\left\{y=\left(y_{1}, y_{2}\right) ; 0<y_{1}<1\right\}$ contains straight lines, and by Proposition 2.2 $A^{p}\left(T_{\Gamma}\right)=\{0\}$. It is easy to compute $K(t)$, and show that it is finite for every $t$. So $A^{p}\left(T_{\Gamma}\right)$ contains non zero functions.

Finally let us restrict to cones: in this case Proposition 2.3 asserts that either the Bergman spaces of $T_{\Gamma}$ and $T_{\Gamma}$ contain both non zero functions, or all these spaces are reduced to $\{0\}$. We can only have a counterexample using Theorem 3 . Let us give the example of a cone in $\mathbb{R}^{3}$ for which there is no constant $C$ such that $K_{c} \leq C K$. Take

$$
\Gamma=\left\{y=\left(y_{1}, y_{2}, y_{3}\right) ; 0<y_{2}<y_{3}, 0<y_{1} y_{3}<y_{2}^{2}\right\} \text {. }
$$

Then

$$
\Gamma^{c}=\left\{y=\left(y_{1}, y_{2}, y_{3}\right) ; 0<y_{1}<y_{2}<y_{3}\right\}
$$


An easy computation gives $K(0)<+\infty$ while $K_{c}(0)=+\infty$, as well as the non existence of a constant $C$.

Remark 3.1. All the results given in this paper are still valid when the Lebesgue measure $d y$ on the cone $\Gamma$ is replaced by some weighted measure $p(y) d y$, under the assumption that $p$ is a measurable positive function which is bounded below on every compact by some strictly positive constant.

\section{REFERENCES}

1. W. Beckner, Inequalities in Fourier Analysis, Ann. of Math. 102 (1975), 159-182.

2. D. Bekolle and A. Temgoua Kagou, Reproducing properties and $L^{p}$ estimates for Bergman projections in Siegel domains of type II, Studia Math. 115 (1995), 219-239.

3. T. G. GENCHEv, Integral representations for functions holomorphic in tube domains, $C . R$. Acad. Bulgare Sci. 37 (6) (1984), 717-720.

4. T. G. Genchev, Paley-Wiener type estimates for functions in Bergman spaces over tube domains, J. Math. Anal. Appl. 118 (1986), 496-501.

5. H. HeINIG and G. SinNamon, Fourier Inequalities and Integral Representations of Functions in Weighted Bergman Spaces over Tube Domains, Indiana Univ. Math. J. 38 (1989), 603-628.

6. L. Hormander, An introduction to complex analysis in several variables (Van Nostrand, Princeton, 1996).

7. A. Koranyi, The Bergman kernel function for tubes over convex cones, Pacific J. Math. 12 (1962), 1355-1359.

8. D. LueCKIng, Closed ranged restriction operators on weighted Bergman spaces, Pacific J. Math. 110 (1984), 145-160.

9. E. M. Stein and G. WeISS, Introduction to Fourier Analysis on Euclidean Spaces (Princeton University Press, Princeton, N.J., 1971).

10. H. TRIEBEL, Theory of function spaces (Birkhaüser, 1983).

11. K. Yosida, Functional Analysis (Springer-Verlag, 1961).

FaCULté des SCIENCES

UNIVERSITÉ DE YAOUNDÉ

BP 812, YAOUNDÉ CAMEROUN

E-mail address: dbekolle@uycdc.uninet.cm
MAPMO - UMR 6628

UNIVERSITÉ D'ORLÉANS

BP 6759, 45067 ORLÉANS CÉDEX 2 FRANCE

E-mail address: bonami@labomath.univ-orleans.fr 